\title{
Acquiring and processing of female EEG signals of various wrist movements for neuro prosthetic applications
}

\author{
Umashankar $\mathbf{G}^{1^{*}}$, Vimala Juliet ${ }^{2}$ and Sheeba Santhosh ${ }^{3}$ \\ ${ }^{1}$ Research scholar, School of Electrical \& Electronics Engineering, Sathyabama Institute of Science \& Technology, Chennai- 119. \\ ${ }^{2}$ Professor, Department of Electronics \& Instrumentation Engineering, SRM Institute of Science \& Technology, Chennai-603203. \\ ${ }^{3}$ Assistant Professor, Department of Electronics \& Communication Engineering, Vel Tech Rangarajan Dr. Sagunthala R\&D Institute of \\ Science and Technology, Chennai. \\ *Corresponding author Email: umashankar.bme@gmail.com
}

\begin{abstract}
Human brain contain neurons which generate electrical signals, this can be recorded through electro encephalograph(EEG). Sensory motor cortices are responsible for motor activity i.e., various body movements, among which wrist movement reveals frequency change in Alpha \& Beta bands of EEG signal. The aim of this approach is to calculate frequency changes responsible for various wrist movements such as flexion, extension, clockwise rotation and anticlockwise rotation, pronation and supination of female in both eyes open and eyes close conditions using FFT, wavelet transform classifier, where the largest set of EEG data is reduced to dimensions and the spectral frequencies for particular wrist movements are classified and the statistical analysis is done of various trials for both eyes open and eyes close conditions in both time domain and frequency domain and the mean and standard deviation of various trials will be compared for eyes open and eyes close condition in both time domain and frequency domain and these values can be implemented for neuro prosthetic applications.
\end{abstract}

Keywords: Electroencephalograph; flexion; Extension; fast fourier transform; classifier.

\section{Introduction}

A brain is the soft convoluted mass of tissue within the skull of vertebrates that function as the coordinating centre of sensation and nervous activity. The brain is the body's chief control centre, containing billions of interconnected nerve cells called neurons. Neurons communicate with each other through electrical signals with respect to internal/external motor/sensory stimuli. A large number of thousands or millions of neurons signals with synchronized activities can be detected on various scalp locations and are called brain signals (measured in cycles per second or Hertz). Electroencephalography (EEG) is an effective modality which helps to acquire brain signals corresponds to human behaviour in terms of motor and sensory states such as, eye movement, lip movement, remembrance, attention, hand clenching etc. from the scalp surface area. These signals are generally categorized as delta, theta, alpha, beta and gamma based on signal frequencies correspond to different types of brain activities which ranges from $0.1 \mathrm{~Hz}$ to more than $100 \mathrm{~Hz}$, Satheesh Kumar, et.al., (2012). However, synchronized activity of a network of neurons shows oscillations at a variety of frequencies. Several of these oscillations have characteristic frequency ranges, spatial distributions associated with different states of brain functions. The neuronal networks underlying some of these oscillations are understood (e.g., the thalamocortical resonance underlying sleep spindles), while many others are not (e.g., the system that generates the posterior basic rhythm). Thereby, it is very difficult to get useful information from these signals directly in the time domain just by observing them. They are basically non-linear and non-stationary in nature. Hence, important features can be extracted for the diagnosis of different diseases using advanced signal processing techniques, Subha D. P, et. al., (2010).

The electrical signal passes through the spinal cord and reaches the muscles that exert the necessary force. Current technology allows collecting and processing these electrical signals from the surface of the scalp area by placing surface electrodes on the scalp, vigneshwari, et.al., (2013). It consists of electrode paste which helps in better conductivity when using a surface electrode. Many systems typically use electrodes, each of which is attached to an individual wire. Some systems use caps or nets into which electrodes are embedded; this is particularly common when highdensity arrays of electrodes are needed. No matter whichever electrode has used the functions succeeding the signal acquisition is same. EEG represents the electrical activities of a brain by means of the graph. EEG have certain bands with separate frequency ranges theta waves varies in the range of $4 \mathrm{~Hz}$ to $7 \mathrm{~Hz}$ and its amplitude generally around $20 \mu \mathrm{V}$, alpha wave varies within the range of 8 to $13 \mathrm{~Hz}$ and about $30-50 \mu \mathrm{V}$ amplitude. For a beta wave, the frequencies vary between $13 \mathrm{~Hz}$ to $30 \mathrm{~Hz}$ and usually have a low voltage between 5-30 $\mu \mathrm{V}$. Different bands carry information about different brain activities, Aowlad Hussain A.B.M, et.al., (2015). After signal acquisition stage, signals are to be pre-processed because the acquired brain signals are most affected by noise and artifacts (unwanted signals). The artifacts that contaminated the EEG signals. To extract the feature matrix from the EEG signal, the artifacts have to be removed. The pre- 
processing step reconstructs the original brain activity by removing contained artifacts. To remove this we apply filtering process. The filters are optimal hence the maximum error between the desired frequency response and the actual frequency response is minimized. After pre-processing the features of EEG signals have to be extracted. Feature extraction is the process of extracting useful information from the signal, Lavanya T.H, et.al., (2010). A variety of different Feature extraction methods exist like Adaptive Auto Regressive parameters (AAR), Fast Fourier Transformations (FFT), PSE, Wavelet Transformations (WT), ICA, padmanabh Mahesh, et. Al., (2015). Extracted EEG signal further classified by using one of the techniques like LDA, QDA, SVM, KNN etc, Khan. Z. H, et. al., (2017).

\section{Materials \& Methods}

\subsection{Electroencephalography}

The term "Electroencephalography" (EEG) is the process of measuring the brain's neural activity as electrical voltage fluctuations along the scalp that results from the current flows in brain's neurons, Mohammad, et.al., (2013). It is not only a function of time; it depends as well on the sensor position on the scalp of the experimental person. This is caused by the localization of the brain activity. Electroencephalography is an electrophysiological monitoring method to record electrical activity of the brain. Electrical recordings can be divided into invasive and noninvasive techniques. Noninvasive recordings are performed by measuring the electrical activity on the patients scalp by means of electrodes. The advantage of noninvasive technique is that it is cheaper, risk free and is easier, Gerrit, et. al., (2016). The system by which EEG electrodes are applied to the head then displayed on EEG recordings is called the International 10-20 system. The International 10-20 system is a standard of measuring the head and placing the electrodes. It depends on four main positions on the head that are easily transferable between the patients. First is the nasion at the bridge of the nose, next is the Inion at the boney prominent at the back of the head and then two Pre-auricular points just anterior to the ear. Each electrode is represented by a number and letter refer fig. 1 The numbers indicate the side of the head so that odd numbers are on the left and even numbers are on the right. In general, lower numbers mean that the electrodes are closer to the mid line, the midline itself is represented by $\mathrm{z}$ (zero). The letters are indicators of the position on head. $\mathrm{F}$ stands for frontal, $\mathrm{C}$ for central, $\mathrm{P}$ for parietal, $\mathrm{T}$ for temporal, $\mathrm{O}$ for occipital. The conventional scalp EEG is used to document the EEG signals. It consists of electrode paste which helps in higher conductivity whilst the use of a floor electrode. Many systems commonly use electrodes. That is connected to a person scalp.

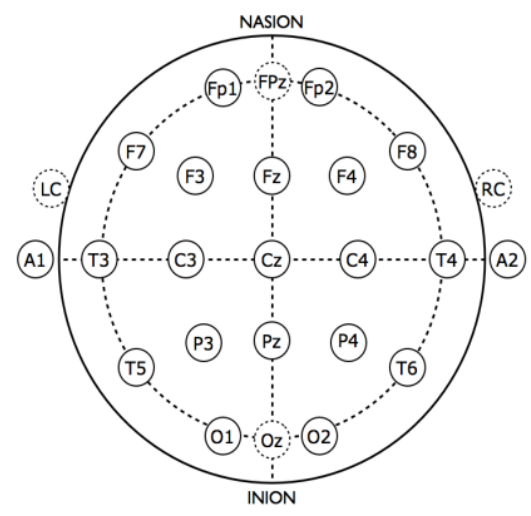

Fig.1: International montage system

\subsection{Matlab}

It is a high-performance language for technical computing. It integrates computation, visualization, and programming in an easy-to-use environment. It is an efficient tool which is used to extract the features and then these feature were processed in the excel application, Ramalingam. T, et. al., (2016). Toolboxes are comprehensive collections of MATLAB functions (M-files) that extend the MATLAB environment to solve classes of problems.

\section{Proposed system}

\subsection{Signal acquisition}

The electrodes are placed according to 10-20 International Montage system. Electrode paste helped the electrode to get secured to the scalp. The individual is asked to execute certain wrist movements and the EEG is recorded simultaneously. Movements include: Clockwise rotation, Anti-clockwise rotation, flexion and extension, Pronation and Supination. Each movement was attempted 20 times, with duration of $12 \mathrm{~s}$ each, in both eyes open and eyes closed condition. The data was later sent and saved in a excel sheet. This procedure was carried for healthy male \& female. This was done to ensure better comparison of EEG. The whole approach is shown in the figure. 2

\subsection{Wavelet transform}

Wavelet transforms are a mathematical means for performing signal analysis when signal frequency varies over time. For certain classes of signals and images, wavelet analysis provides more precise information about signal data than other signal analysis techniques. The basic function consists of wavelets, every one being a scaled and transformed version of identical wave. The wave transform makes it potential to study a characteristic in numerous scales at the same time. This is the most advantageous feature of wavelet transformation. Adding there to, the amount of resolution depends on the size, Lung Chuin Cheong, et. al., (2015).

\subsection{Daubechies wavelet transform}

The dbN wavelets are the Daubechies' extremal phase wavelets. $\mathrm{N}$ refers to the number of vanishing movements. The wavelets are characterized by a maximal number of vanishing movements for a given support. With each wavelet type of this class, there is a scaling function, which generates an orthogonal multi resolution. Each set of original data is fed as input say N; the wavelet function will be applied to calculate N/2. The steps included in iteration are calculation of scaling factor and wavelet function value. Every time the iteration continues by upgrading the value of $i$.

\subsection{Wavelet decomposition and reconstruction}

If we glance at what wavelet decomposition does, it breaks down a signal into a high and a lower part oppressing two filters and decimates by an element of two. It then repeats this on the approximation part (low) for every level you decompose. Thus, To decompose at one level, the signal could pass through each filter and decimate the result by two at the top. This preserves the number of total samples. an equivalent logic applies to following and ulterior levels. The whole procedure is inversed for wavelet reconstruction, Kumar. N, et al., (2017). 


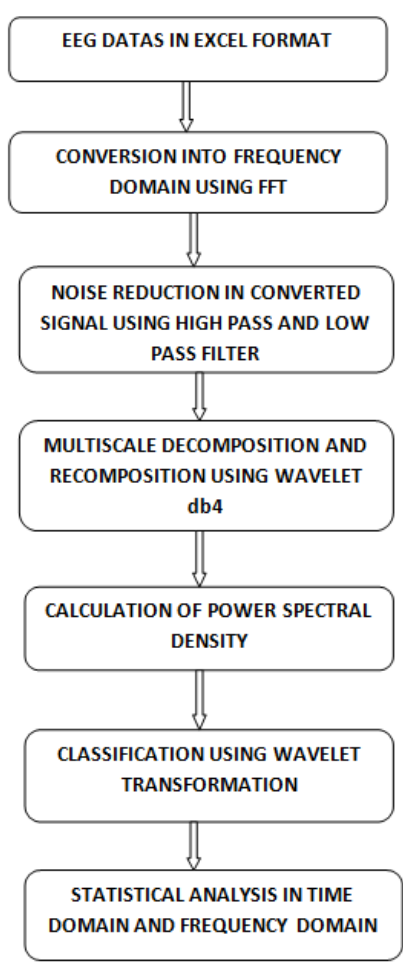

Fig. 2: Overall block diagram

\subsection{Power Spectral Density}

The power spectral density (PSD) is intended for continuous spectra. The integral of the PSD over a given frequency band computes the average power in the signal over that frequency band. The range of the frequency vector depends on the SpectrumType value. (PSD) is the frequency response of a random or periodic signal. It tells us where the average power is distributed as a function of frequency.

\subsection{Statistical Analysis}

Standard deviation it is the measure of how spread out the data is. A low SD denotes that the data points tend to be close to mean thus are not wide spread, while the high SD values indicates less closeness to mean and thus the data is wide spread.

Mean it is the foremost common and best general-purpose measure of the mid-point (around which all other values cluster) of a set of values, however is prone to distortion by the presence of extreme values and may need use of a measure of distortion (such as deviation or standard deviation). Additionally, known as arithmetic mean

Median it is the value separating the higher half of a data sample, a population, or a probability distribution, from the lower half. For a data set, it may be thought of as the "middle" value. The median is a commonly used measure of the properties of a data set in statistics and probability theory.

Mode of a set of data values is the value that appears most often. It is the value $\mathrm{x}$ at which its probability mass function takes its maximum value. In other words, it is the value that is most likely to be sampled.

\section{Results And Discussion}

EEG signals are obtained in Excel Format as shown in figure 3, and the Excel file for a particular movement is loaded as input in MATLAB program. Time period for each trial is about 10 seconds.

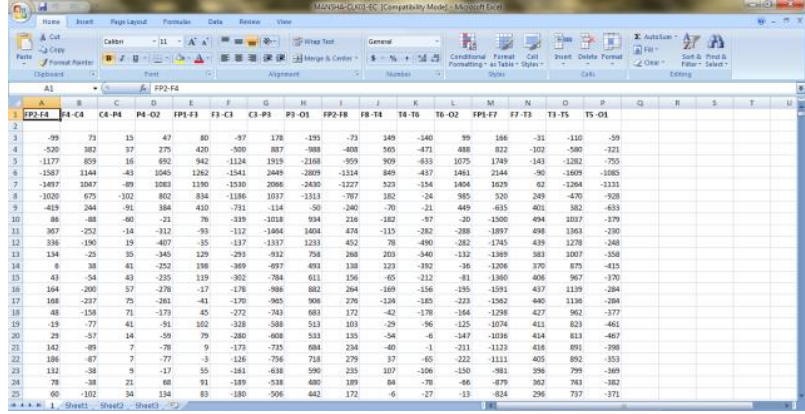

Fig. 3: Input in Excel format

To have a better analysis of EEG signal, the signal is converted to frequency domain refer figure 4 . The reason behind using FFT in this approach is, FFT is a simpler for EEG signals when the value of $\mathrm{N}$ is large. Basically, any time-dependent signal will be broken down in collection of sinusoids. In this way, prolonged and noisy EEG recordings will be conveniently plotted in a frequency power-spectrum. By doing so, hidden feature swill become apparent. By adding all the sinusoids up after FFT, the original signal will be restored, thus no data is lost.

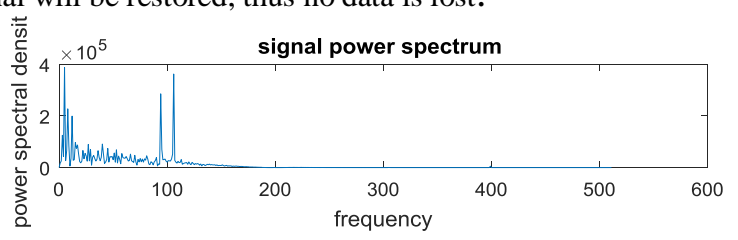

Fig. 4: EEG Power spectrum

EEG signals are extremely weak and affected by different types of noises and impairments that need to be carefully eliminated. Even though EEG signals are filtered during acquisition using notch filter; notch is a very selective filter with a very high rejection just for a tiny frequency band around the selected frequency. It will not attenuate other frequencies which belong to the EEG signal. So band pass filter is used additionally in the program refer figure 5 . But it is very difficult to design a single band pass filter that can selectively eliminate the noises affecting the EEG. The filtering is accomplished by using multiple filters with specific purposes. So, both High pass filter and Low pass filter is used. The filtering is accomplished by using multiple filters with specific purposes.
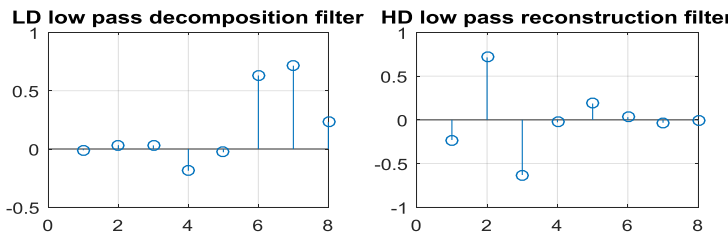

LR high pass decomposition filter HR high pass reconstruction filter
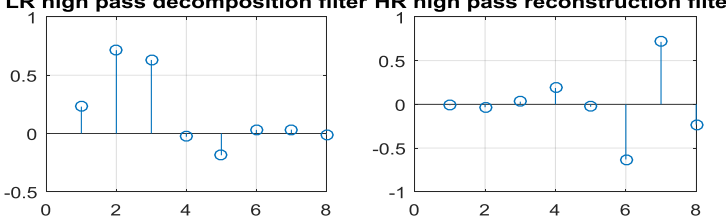

Fig. 5: Filtered EEG signal

The purpose of using multilevel wavelet decomposition in this approach is because multilevel wavelet decomposition provides the knowledge regarding frequency elements present and enhances the information about the signal for further processing. Wavelet decomposition breaks down the EEG signal into a high and a low part in pattern of to filters thus decimates by a component of two, which is shown in the figure 6 . It then repeats this on the approximation part (low) for each level of decomposition. Therefore if the signal is decomposed at one level, then the signal is just passed through every filter and decimates the result by two 
at the highest. This preserves the quantity of total samples. An equivalent logic applies to following and ulterior levels.

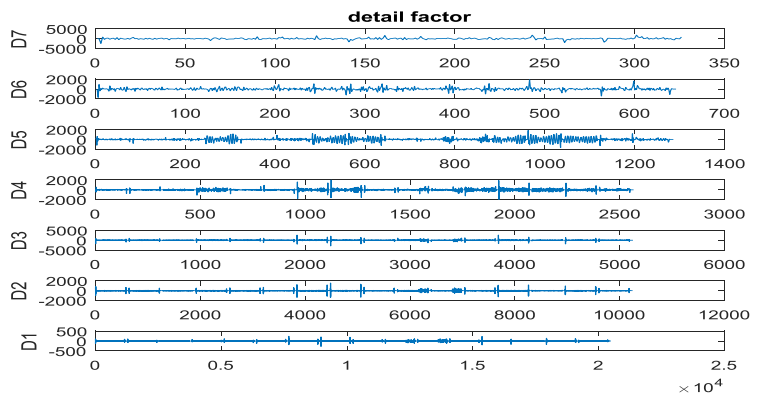

Fig. 6: Wavelet Decomposition

During reconstruction, detail coefficients and approximation coefficients at every stage are up sampled by two, passed by high and low pass synthesis filters and addedlater. To get the original signal, the method is continued by similar number of steps like decomposition method. The purpose of using Daub4 wavelet scaling is to illustrate how different types of signals are optimized for different scaling factors figure 7 (2, 4, 8, 16, 32, and 64).

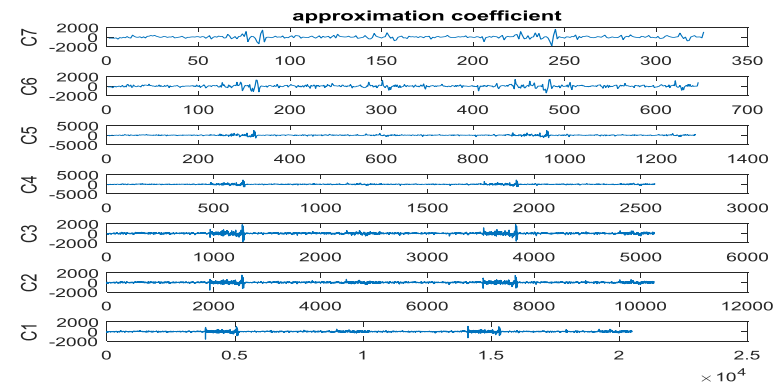

Fig. 7: Wavelet Recomposition

Power Spectral Density is calculated to find where the frequency variation is very high, and the frequency variation is very low. It reflects the 'frequency content' of the signal or the distribution of signal power over frequency. Peak value in the output image figure 8 denotes the corresponding frequency for the particular wrist movement.

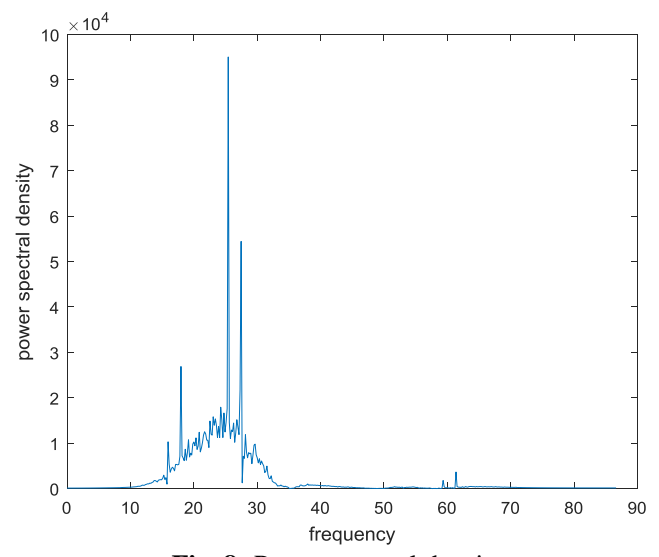

Fig. 8: Power spectral density

Comparison of eyes close and eyes open condition of all the wrist movements alongwith its statistical analysis such as frequency domain and time domain are shown in below.

ANTICLOCKIVISE

EYES CLOSE

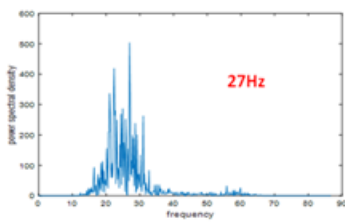

ANTICLOCKWISE EYES CLOSE

FRE DOMAIN ANALYSIS

\begin{tabular}{|l|l|l|}
\hline Min & 0 & 0.0023126 \\
\hline Max & 86.64 & 2147.78 \\
\hline Mean & 43.32 & 27.746 \\
\hline Median & 43.32 & 2.6048 \\
\hline Mode & 0 & 0.0023126 \\
\hline $\begin{array}{l}\text { Standard } \\
\text { Deviation }\end{array}$ & 25.08 & 172.158 \\
\hline Range & 86.64 & 2147.78 \\
\hline
\end{tabular}

TIME DOMAIN ANALYSIS

ANTICLOCKWISE EYES OPEN

FRE DOMAIN ANALYSIS

\begin{tabular}{|l|r|}
\hline Average & -0.50059 \\
\hline $\begin{array}{l}\text { Standard } \\
\text { deviation }\end{array}$ & 64.35295 \\
\hline kurtosis & 31.27593 \\
\hline
\end{tabular}

TIME DOMAIN ANALYSIS

\begin{tabular}{|l|l|l|}
\hline Min & 0 & 0.0118145 \\
\hline Max & 86.64 & 13857.08 \\
\hline Mean & 43.32 & 26.976 \\
\hline Median & 43.32 & 3.8118 \\
\hline Mode & 0 & 0.0118145 \\
\hline $\begin{array}{l}\text { Standard } \\
\text { Deviation }\end{array}$ & 25.08 & 732.718 \\
\hline Range & 86.64 & 13857.08 \\
\hline
\end{tabular}

\begin{tabular}{|l|r|}
\hline Average & -0.49934 \\
\hline $\begin{array}{l}\text { Standard } \\
\text { deviation }\end{array}$ & 414.6078 \\
\hline kurtosis & 9.85541 \\
\hline
\end{tabular}

Fig. 9: Anticlockwise movement statistical analysis

The figure 9 shows the frequency data analysis and time data analysis for anti-clockwise rotation for female in eyes close and eyes open condition. Frequency data analysis is done for PSD after processing of signal takes place in frequency domain. Time data analysis is done for raw EEG signal without processing or filtration takes place in time domain. The mean value i.e. the frequency value for this particular movement is observed to be 27.746 in eyes close and 26.976 in eyes open condition. Whereas in time domain analysis the mean value is observed to be -0.50059 in eyes close and -0.49934 in eyes open condition. The average spread out of data i.e. SD of the Frequency analysis is 172.158 in eyes close and 732.718 in eyes open condition and for time domain is 64.352 in eyes close and 414.60 in eyes close condition.

The figure 10 shows the frequency data analysis and time data analysis for anti-clockwise rotation for female in eyes close and eyes open condition. Frequency data analysis is done for PSD after processing of signal takes place in frequency domain. Time data analysis is done for raw EEG signal without processing or filtration takes place in time domain. The mean value i.e. the frequency value for this particular movement is observed to be 21.516 in eyes close and 25.763 in eyes open condition. Whereas in time domain analysis the mean value is observed to be -0.50025 in eyes close and -0.49949 in eyes open condition. The average spread out of data i.e. SD of the Frequency analysis is 1427.798 in eyes close and 596.176 in eyes open condition and for time domain is 140.9393 in eyes close and 93.08799 in eyes close condition.

The figure 11 shows the frequency data analysis and time data analysis for anti-clockwise rotation for female in eyes close and eyes open condition. Frequency data analysis is done for PSD after processing of signal takes place in frequency domain. Time data analysis is done for raw EEG signal without processing or filtration takes place in time domain. 
CLOCKUVISE

EYES CLOSE
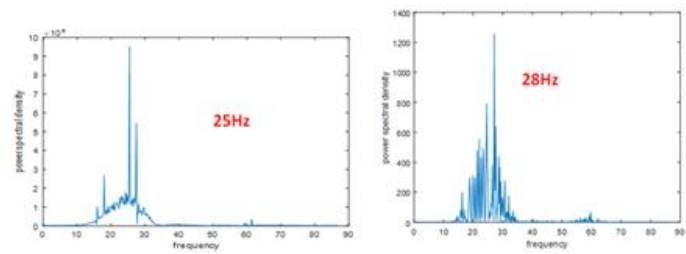

CLOCKWISE EYES CLOSE

FRE DOMAIN ANALYSIS

\begin{tabular}{|l|l|l|}
\hline Min & 0 & 1.729063 \\
\hline Max & 86.64 & 21354.34 \\
\hline Mean & 43.32 & 21.516 \\
\hline Median & 43.32 & 62.4678 \\
\hline Mode & 0 & 1.729063 \\
\hline $\begin{array}{l}\text { Standard } \\
\text { Deviation }\end{array}$ & 25.08 & 1427.798 \\
\hline Range & 86.64 & 21354.34 \\
\hline
\end{tabular}

TIME DOMAIN ANALYSIS

CLOCKUISE EYES OPEN

FRE DOMAIN ANALYSIS

\begin{tabular}{|l|l|l|}
\hline Min & 0 & 0.0034151 \\
\hline Max & 86.64 & 10768.5 \\
\hline Mean & 43.32 & 25.763 \\
\hline Median & 43.32 & 2.71314 \\
\hline Mode & 0 & 0.0034151 \\
\hline $\begin{array}{l}\text { Standard } \\
\text { Deviation }\end{array}$ & 25.08 & 596.176 \\
\hline Range & 86.64 & 10768.5 \\
\hline
\end{tabular}

TIME DOMAIN ANALYSIS

Fig. 10: clockwise movement statistical analysis

The mean value i.e. the frequency value for this particular movement is observed to be 29.712 in eyes close and 26.627 in eyes open condition. Whereas in time domain analysis the mean value is observed to be -0.49932 in eyes close and -0.4995 in eyes open condition. The average spread out of data i.e. SD of the Frequency analysis is 112.976 in eyes close and 112.976 in eyes open condition and for time domain is 371.94 in eyes close and 422.134 in eyes close condition

FLEXION \& EXTENSION

EYES CLOSE
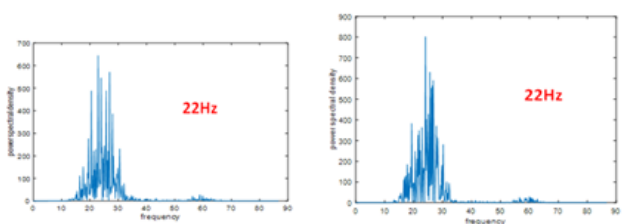

FLEXION \& EXTENSION EYES CLOSE

FRE DOMAIN ANALYSIS

\begin{tabular}{|l|l|l|}
\hline Min & 0 & 0.001813 \\
\hline Max & 86.64 & 1283.7 \\
\hline Mean & 43.32 & 29.712 \\
\hline Median & 43.32 & 0.99778 \\
\hline Mode & 0 & 0.001813 \\
\hline $\begin{array}{l}\text { Standard } \\
\text { Deviation }\end{array}$ & 25.08 & 112.976 \\
\hline Range & 86.64 & 1283.7 \\
\hline
\end{tabular}

TIME DOMAIN ANALYSIS

FLEXION \& EXTENSION EYES OPEN

FRE DOMAIN ANALYSIS

\begin{tabular}{|l|l|l|}
\hline Min & 0 & 0.001813 \\
\hline Max & 86.64 & 1283.7 \\
\hline Mean & 43.32 & 26.627 \\
\hline Median & 43.32 & 0.99778 \\
\hline Mode & 0 & 0.001813 \\
\hline $\begin{array}{l}\text { Standard } \\
\text { Deviation }\end{array}$ & 25.08 & 112.976 \\
\hline Range & 86.64 & 1283.7 \\
\hline
\end{tabular}

TIME DOMAIN ANALYSIS

\begin{tabular}{|l|r|}
\hline Average & -0.4995 \\
\hline $\begin{array}{l}\text { Standard } \\
\text { deviation }\end{array}$ & 422.1347 \\
\hline kurtosis & 35.77343 \\
\hline
\end{tabular}

Fig. 11: Flexion \& Extension movement statistical analysis
PRONATION \& SUPINATION

EYES CLOSE

EYES OPEN
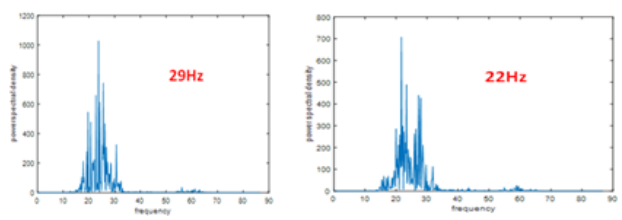

PRONATION \& SUPINATION EYES CLOSE

FRE DOMAIN ANALYSIS

TIME DOMAIN ANALYSIS

\begin{tabular}{|l|l|l|}
\hline Min & 0 & 0.00779 \\
\hline Max & 86.64 & 3119.92 \\
\hline Mean & 43.32 & 24.198 \\
\hline Median & 43.32 & 1.34838 \\
\hline Mode & 0 & 0.0077942 \\
\hline $\begin{array}{l}\text { Standard } \\
\text { Deviation }\end{array}$ & 25.08 & 225.63 \\
\hline Range & 86.64 & 3119.92 \\
\hline
\end{tabular}

\begin{tabular}{|l|r|}
\hline Average & -0.49985 \\
\hline $\begin{array}{l}\text { Standard } \\
\text { deviation }\end{array}$ & 410.7045 \\
\hline kurtosis & 13.54853 \\
\hline
\end{tabular}

PRONATION \& SUPINATION EYES OPEN

FRE DOMAIN ANALYSIS

TIME DOMAIN ANALYSIS

\begin{tabular}{|c|c|c|c|c|}
\hline Min & 0 & 0.0120302 & \multirow{7}{*}{\begin{tabular}{|l} 
Average \\
Standard \\
deviation \\
kurtosis \\
\end{tabular}} & \multirow[b]{4}{*}{-0.50061} \\
\hline Max & 86.64 & 947.8 & & \\
\hline Mean & 43.32 & 25.402 & & \\
\hline Median & 43.32 & 1.12278 & & \\
\hline Mode & 0 & 0.0120302 & & \\
\hline $\begin{array}{l}\text { Standard } \\
\text { Deviation }\end{array}$ & 25.08 & 92.328 & & 572.5202 \\
\hline Range & 86.64 & 947.8 & & 60.80827 \\
\hline
\end{tabular}

Fig. 12: Pronation \& Supination movement statistical analysis

The figure 12 shows the frequency data analysis and time data analysis for anti-clockwise rotation for female in eyes close and eyes open condition. Frequency data analysis is done for PSD after processing of signal takes place in frequency domain. Time data analysis is done for raw EEG signal without processing or filtration takes place in time domain. The mean value i.e. the frequency value for this particular movement is observed to be 24.196 in eyes close and 25.402 in eyes open condition. Whereas in time domain analysis the mean value is observed to be -0.49985 in eyes close and -0.50061 in eyes open condition. The average spread out of data i.e. SD of the Frequency analysis is 225.63 in eyes close and 92.326 in eyes open condition and for time domain is 410.704 in eyes close and 572.52 in eyes close condition.

\section{Conclusion}

This approach proposed that EEG frequencies of female for various wrist movements such as flexion and extension, anticlockwise rotation and clockwise rotation, pronation and supination have been extracted in both eyes open and eyes close condition through $10-20$ electrode system. The obtained frequencies are almost similar to that of the alpha and beta rhythms of brain signals, i.e the frequencies lies between 22 and $28 \mathrm{~Hz}$. From the observed frequency range beta waves are more prominent during these movements. Since the variations of frequency for each movement is very small, it can be analyzed through the classification techniques. These processed frequencies can be used for neuro prosthetic applications.

\section{References}

[1] AowladHossain. A.B.M, WasiurRahman. Md, ManjurulAhsanRiheen. Left and Right Hand Movements EEG Signals Classification Using Wavelet Transform and Probabilistic Neural Network. International Journal of Electrical and Computer Engineering (IJECE) Vol. 5 (1), 2015, pp: 92-101.

[2] Gerrit Lange, Cheng Yee Low, Khairunnisa, Farah AktharHanapiah, FadhlanKamaruzaman. Classification of Electroencephalogram Data from Hand Grasp and Release Movements for BCI Controlled Prosthesis. Procedia Technology. vol 26, 2016, pp: $374-381$. 
[3] Khan Z.H, Nasir Hussain, Mohsin. I, Tiwana. Classification of EEG signals for wrist and grip movements using echo state network. Vo 28(3), 2017, pp: 1095-1102.

[4] Kumar. N, Alam. K, Siddique. A. H. Wavelet Transform For Classification Of EEG Signal Using SVM and ANN. Biomed pharmacology Journal, Vol 10(4), 2017, pp: 2061-2069

[5] Lavanya. T. H, Jyothi. K. S. EEG Based Classification of Hand Movements using BCI. Vol. 5(4), 2016,pp: 687 - 691.

[6] Lung Chuin Cheong, RubitaSudirman and SitiSurayaHussin. Feature extraction of EEG signal using wavelet transform for autism classification. vol.10, 2015, pp: 8533 - 8540.

[7] Mohammad. H, Alomari, AyaSamaha, KhaledAlKamha. Automated Classification of L/R Hand Movement EEG Signals using Advanced Feature Extraction and Machine Learning. Vol. 4(6), 2013, pp: 207 212.
[8] Padmanabh Mahesh, Shastri. R. K, Biradar. S. D. EEG signal processing techniques for mental task classification, Vol 2(1), 2015 pp: 66-74.

[9] Ramalingam. V, Mohan. S, Rebecca JeyaVadhanam. B, Sugumaran.V. Classification of EEG Signals for Prosthetic Limb Movements with ARMA Features Using C4.5 Decision Tree Algorithm. Indian Journal of Science and Technology.Vol 9(47), 2016, pp:1-11.

[10] Satheesh Kumar. J, Bhuvaneswari. P. Analysis of electrencephalography(EEG) signal and its characterisation - A study in Procedia Engineering. Vol 38 , 2012 ,pp: 2525 - 2536

[11] Subha D. P, Joseph P.K, Acharya U R, Lim CMEEG signal analysis: a survey in J Med Syst. Vol 34(2), 2010, pp: 195-212.

[12] Vigneshwari. C, Vimala. V, VairaVignesh. S, Sumithra. G. Analysis of Finger Movements Using EEG Signal. International Journal of Emerging Technology and Advanced Engineering Vol 3 (1), 2013 pp: 